\title{
Suplementação de antioxidantes: enfoque em queimados
}

\author{
Antioxidant supplementation: \\ focusing on burned patients
}

Eliana BARBOSA ${ }^{1}$

Emília Addison Machado MOREIRA²

Joel FAINTUCH ${ }^{3}$

Maurício José Lopes PEREIMA ${ }^{1,4}$

Na queimadura, os radicais livres são produzidos durante a reposição de fluídos, alterando numerosos componentes, como os ácidos nucléicos, os lipídios e as proteínas. Sendo assim, se, por um lado, essa restauração do fluxo sanguíneo é, em geral, necessária para recuperar a função celular normal, por outro lado a reintrodução do sangue oxigenado nos tecidos isquêmicos contribui para uma produção excessiva de radicais livres derivados do oxigênio, exacerbando a lesão mediada pela isquemia. O papel dos neutrófilos ativados na célula após a queimadura também tem recebido atenção especial. A partir do sistema NADPH oxidase os neutrófilos aderidos podem produzir uma explosão de radical superóxido, produzindo um dano tecidual importante. A queimadura, além de aumentar a produção de radicais livres, também é capaz de impedir os mecanismos de defesa antioxidante, submetendo o paciente queimado ao estresse oxidativo, o qual se constitui em fator perpetuante da resposta inflamatória sistêmica, levando à piora progressiva do estado metabólico. Com esses dados, objetiva-se revisar os principais estudos com a suplementação de antioxidantes em pacientes críticos, com ênfase em pacientes queimados, e descrever os possíveis benefícios dessa intervenção.

Termo de indexação: antioxidantes; estresse oxidativo; queimaduras; radicais livres.

\section{A B S T R A C T}

In burn injuries, free radicals are produced during fluid replacement, altering multiple components of the organism such as nucleic acids, lipids and proteins. Thus, if on the one hand restoring blood flow is generally necessary to recover normal cell function, on the other hand oxygen reperfusion into ischemic tissues triggers

\footnotetext{
1 Hospital Infantil Joana de Gusmão, Unidade de Queimados. R. Barbosa, 152, Agronômica, 88025-301, Florianópolis, SC, Brasil. Correspondência para/Correspondence to: E. BARBOSA. E-mail: <elianabarbosa@myhimax.com.br>.

2 Universidade Federal de Santa Catarina, Programa de Pós-Graduação em Nutrição. Florianópolis, SC, Brasil.

3 Universidade de São Paulo, Faculdade de Medicina, Departamento de Gastroenterologia. São Paulo, SP, Brasil.

${ }^{4}$ Universidade Federal de Santa Catarina, Centro de Ciências da Saúde, Departamento de Pediatria. Florianópolis, SC, Brasil.
} 
$694 \mid$ E. BARBOSA et al.

massive production of oxygen free radicals, therefore further exacerbating ischemic injury. The role of neutrophiles activated in the cell after burns has also received special attention. By means of the NADPH-oxidase system, adherent neutrophiles may release superoxide radicals inflicting major tissue damage. The burn injury not only increases the production of free radicals but it is also capable of impairing the antioxidant defense mechanism, subjecting the burned patient to oxidative stress, which can be a perpetuating factor of the inflammatory response and lead to progressive metabolic deterioration. Therefore, the purpose of this work is to provide updated information on antioxidant supplementation in burned patients and describe the benefits of this intervention.

Indexing terms: antioxidants; oxidative stress; burns; free radicals.

\section{N T R O D U ÇÃ O}

O trauma por queimadura é a segunda causa de morte em crianças menores de cinco anos de idade e a causa mais comum de morte acidental no ambiente domiciliar ${ }^{1}$. Logo após o trauma térmico, há um aumento acentuado da taxa de metabolismo basal, que se mantém elevada por períodos prolongados. Em crianças, essa taxa metabólica aumenta linearmente com a extensão da superfície corporal queimada, podendo chegar a 150\% e mesmo 200\% acima das necessidades nutricionais, supostamente com o intuito de manter a temperatura do corpo, sintetizar novos tecidos e equilibrar o hiperdinamismo que se instala².

Na queimadura ocorre a destruição da barreira epitelial e da microbiota residente na pele, rompendo o seu efeito protetor. A presença de tecido desvitalizado, de proteínas degradadas e a queda do suprimento de oxigênio favorecem a proliferação de microorganismos patógenos do ambiente, da pele normal que circunda a lesão, ou da própria lesão. Essa condição, aliada a uma importante deficiência imunológica, pode ocasionar a geração de um foco infeccioso e, posteriormente, sepse ${ }^{3}$. Como conseqüência do processo infeccioso, uma complexa rede de respostas ocorre de forma heterogênea na criança queimada.

Em resposta a estímulos específicos - tais como bactérias ou fragmentos bacterianos e espécies moleculares como agregados de imunoglobulinas (lgG), componentes polimorfos da interleucina 1 (IL-1) - é iniciada uma seqüência complexa de eventos denominada fagocitose. A fagocitose é um processo dependente de energia, na qual o oxigênio é rapidamente consumido, ocorrendo uma "explosão respiratória" e produção de radicais livres 4 .

Além disso, após grandes queimaduras, a correta reposição agressiva de volume de líquido intra e/ou extracelular na fase inicial é de extrema importância para evitar o choque hipovolêmico e a insuficiência e necrose tubular aguda. Dessa forma, restaura-se o volume intra-vascular, perfundindo os tecidos e aumentando a sobrevida desses pacientes ${ }^{5}$. Porém, essa reintrodução do sangue oxigenado nos tecidos isquêmicos contribui para a produção de um excesso de radicais livres derivados do oxigênio, que, por sua vez, produzem danos adicionais ao tecido ${ }^{6,7}$.

Assim, a compreensão dos mecanismos envolvidos na produção excessiva de radicais livres em mecanismos fisiológicos, como fagocitose, reação inflamatória e fenômeno de isquemia/reperfusão, em indivíduos que sofreram queimaduras, é de suma importância para uma terapêutica adequada.

Dessa maneira, qualquer substância, seja ela um produto natural, composto sintético ou fármaco, que demonstre estimular as defesas antioxidantes ou diminuir a produção de radicais livres, constitui-se como importante objeto de estudo para o tratamento ou prevenção de doenças.

Diante do exposto, objetiva-se revisar os principais estudos com a suplementação de antioxidantes em pacientes críticos, com ênfase em pacientes queimados, com a finalidade de descrever os possíveis benefícios dessa intervenção. 


\section{QUEIMADURA E ESTRESSE OXIDATIVO}

Na queimadura, os radicais livres (RL) são produzidos durante a reposição de fluídos alterando numerosos componentes, incluindo ácidos nucléicos, lipídios e proteínas ${ }^{6,7}$. Este processo ocorre durante a isquemia, na qual a xantina oxidase $(\mathrm{XO})$ e a hipoxantina encontram-se com suas concentrações aumentadas, de modo que, quando o oxigênio molecular é reintroduzido durante a reperfusão, a XO cataliza sua conversão a superóxido. Essa produção aumentada de superóxido, suprimindo a capacidade de defesa das enzimas endógenas, contribui para aumentar o estresse oxidativo e causar lesão celular7,8.

O papel dos neutrófilos ativados após a queimadura também tem recebido atenção especial. A partir da mobilização do sistema adenina difosfato nicotinamida reduzido (via NADPH oxidase), os neutrófilos aderidos ao endotélio podem produzir uma explosão de radical superóxido?.

Efetivamente, acredita-se que, em grande parte, a lesão celular e endotelial seja amplificada pela liberação de radicais livres e enzimas dos neutrófilos. Uma vez firmemente aderidos à célula endotelial, os neutrófilos criariam um microambiente, que permitiria a alta concentração de agentes lesivos. Além de sintetizarem prostaglandinas, os neutrófilos liberam espécies reativas adicionais, radicais livres e enzimas proteolíticas, em particular a elastase, expandindo as lesões teciduais. Outra enzima encontrada nos neutrófilos é a mieloperoxidase, a qual catalisa a reação do peróxido de hidrogênio com cloro, formando o ácido hipocloroso $(\mathrm{HClO})$, outro potente agente oxidante ${ }^{6}$.

Um número crescente de estudos confirma o envolvimento das espécies reativas de oxigênio (EROs) na destruição térmica9-12. A literatura tem sugerido que os oxidantes gerados contribuem para danos locais adicionais contribuindo na formação do edema. A ativação do sistema complemento, por sua vez, resultaria na inflamação de órgãos à distância ${ }^{13,14}$. Também a defesa antioxidante estaria enfraquecida nesse contexto ${ }^{15}$.

O desequilíbrio entre moléculas oxidantes e antioxidantes que resulta na indução de danos celulares pelos radicais livres, tem sido chamado de estresse oxidativo ${ }^{16}$, gerando danos a lipídios, proteínas, carboidratos e ácidos nucléicos e, finalmente, morte celular ${ }^{17}$. O estresse oxidativo pode levar a uma alteração nos sistemas antioxidantes ao induzir ou reprimir proteínas que participam desses sistemas, ou ao esgotar as reservas celulares de substâncias antioxidantes ${ }^{18}$.

Uma das conseqüências mais estudadas do estresse oxidativo é a peroxidação lipídica, que constitui uma reação em cadeia nos ácidos graxos polinsaturados das membranas celulares, alterando a permeabilidade, fluidez e integridade das mesmas $^{19,20}$. Os produtos principais dessa degradação compreendem os derivados da decomposição de hidroperóxidos como álcoois, aldeídos, cetonas e outros hidrocarbonetos. A dosagem do malondialdeído (MDA), utilizando-se o teste do ácido tiobarbitúrico (TBA), serve de indicador para a lipoperoxidação ${ }^{21}$.

A queimadura, com seus componentes de necrose celular, infecção e ativação citoquímica, está primariamente caracterizada pelo desenvolvimento de uma reação inflamatória, induzindo uma resposta de fase aguda intensa. Também a produção de radicais livres está associada com inflamação, e peróxidos lipídicos circulantes têm sido mostrados aumentados em pacientes queimados durante a primeira semana $a^{9,12}$.

Alguns estudos têm avaliado o estado antioxidante sérico total em grupos de pacientes graves, relatando níveis elevados de peróxidos lipídicos e redução expressiva nas defesas antioxidantes, evidenciando o estresse oxidativo ${ }^{19,22,23}$. Uma diminuição de antioxidantes lipídicos ( $\alpha$-tocoferol, $\beta$-caroteno, vitamina A) e aumento da peroxidação lipídica também foram observados durante 30 dias em pacientes queimados, mesmo nos que exibiam apenas $10 \%$ de superfície corporal queimada ${ }^{14}$. 
696 | E. BARBOSA et al.

Esses dados, coletivamente, sustentam a hipótese de que o estresse oxidativo é um ponto crítico na cascata nociva mediada pela queimadura, e sugerem que estratégias antioxidantes direcionadas para inibição da produção ou seqüestro de radicais livres possam exercer um efeito protetor?

Entre os principais antioxidantes têm-se os enzimáticos e os não enzimáticos. Entre os antioxidantes enzimáticos celulares encontram-se as enzimas superóxido dismutase (SOD), a catalase (CAT) e a glutationa peroxidase (GPx), que provêem a primeira linha de defesa endógena ${ }^{24}$. A ação combinada desses três antioxidantes fisiológicos mantém a célula com concentrações reduzidas de $\mathrm{O}_{2} \cdot$ - (radical superóxido) e $\mathrm{H}_{2} \mathrm{O}_{2}$ (peróxido de hidrogênio), evitando a geração de - $\mathrm{OH}$ (radical hidroxila) (pela reação de Haber Weiss). Essa é uma estratégia comum a todos os organismos aeróbicos ${ }^{25}$.

Dos componentes não enzimáticos da defesa antioxidante destacam-se alguns minerais (zinco, selênio e ferro), vitaminas (ácido ascórbico, vitamina $\mathrm{E}$, vitamina $\mathrm{A}$, riboflavina), carotenóides (beta-caroteno, licopeno e luteína) e bioflavonóides (genisteína, quercetina, extrato de ginkgo biloba) ${ }^{26,27}$

Sendo assim, a manipulação dos níveis plasmáticos desses antioxidantes, exógenos ou endógenos, seja por meio de suplementos na dieta ou por estímulo de suas reações de síntese, constitui um importante tema de pesquisa.

\section{S U PLE M E N T A ÇÃ O DE ANTIOXIDANTES}

Em se tratando de suplementação de antioxidantes, a literatura é escassa quando se refere especificamente a paciente queimado. Sendo assim, dados de estudos em pacientes criticamente doentes foram incluídos na comparação do efeito dos antioxidantes no estresse oxidativo, uma vez que o indivíduo queimado inclui-se nessa categoria, dado o estado catabólico imposto pela queimadura ${ }^{28}$. A deficiência de micronutrientes nesses pacientes, especificamente, selênio, cobre e zinco, reduz a resistência a vários microorganismos e aumenta a taxa de infecção pelas bactérias, fungos, parasitas e vírus ${ }^{18,29}$. A imunidade é afetada por dois fatores: alterações das defesas não específicas, incluindo a barreira física (ruptura da pele), produção de citoquinas, fagocitose e produção de complemento, além de alterações da resposta antígeno - específica na produção de anticorpos e imunidade celular ${ }^{30}$.

Segundo Berger \& Chiolero $^{10}$, grandes queimaduras induzem a uma severa depressão nos níveis de cobre, zinco e selênio, especialmente na primeira semana após o acidente. Estudos têm mostrado que os níveis séricos podem ser restaurados com a suplementação de elementos traços em quantidades de 6 a 8 vezes acima daquela recomendada pela RDA (Recommended Dietary

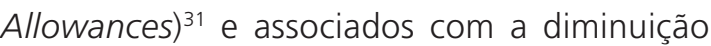
significativa de infecções pulmonares e menor tempo de internação $0^{29,32}$.

Em um recente estudo analisando o estado de micronutrientes em crianças queimadas, verificou-se que as médias plasmáticas de zinco e cobre estavam baixas e a excreção urinária dos mesmos acima do normal, na admissão e alta hospitalar. Esses resultados foram encontrados apesar de as quantidades dietéticas de ambos os micronutrientes estarem em torno de três vezes a RDA preconizada pela DRI (Dietary Reference Intakes) ${ }^{33}$, sugerindo que os mesmos foram compensados inadequadamente durante a hospitalização 34 .

Agay et al. ${ }^{35}$, pesquisando as alterações de elementos traços antioxidantes no plasma em ratos queimados, observaram uma diminuição significativa do selênio e do zinco séricos no primeiro dia após o trauma, sendo este último associado com o aumento da atividade da superóxido dismutase (SOD) e do zinco hepáticos. A atividade da glutationa peroxidase (GPx) no primeiro dia apresentou uma diminuição significativa no plasma e uma diminuição moderada no fígado, sendo que o selênio aumentou significativamente 
no rim, no qual a GPx é sintetizada. Os autores sugerem que essas alterações podem estar refletindo um mecanismo adaptativo para neutralizar os efeitos deletérios das desordens metabólicas e do estresse oxidativo associado com a queimadura. Em conseqüência, a suplementação precoce combinada de antioxidantes deveria ser considerada em pacientes queimados.

Na síndrome da resposta inflamatória

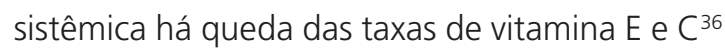
e valores baixos de vitamina $C$ são preditivos de disfunção múltipla de órgãos e sistemas em populações de risco ${ }^{19}$.

Assim, um aumento na ingestão dessas vitaminas e de elementos traços seria aconselhável para diminuir a peroxidação lipídica29,37.

$\mathrm{O}$ sinergismo entre as vitaminas $\mathrm{C}$ e E é, freqüentemente, mencionado na literatura, mostrando que a interação dessas vitaminas é efetiva na inibição da peroxidação dos lipídios da membrana, na proteção do DNA ${ }^{38}$ e na diminuição da incidência de complicações infecciosas ${ }^{39,40}$. Conseqüentemente a modulação da peroxidação lipídica se tornou um importante ponto de debate, e antioxidantes endógenos e exógenos têm sido usados para diminuir esse fenômeno ${ }^{10,23,41}$.

As evidências de estresse oxidativo, lesão tecidual e indução da resposta inflamatória sistêmica fornecem razões biológicas para suplementação de antioxidantes em pacientes $\operatorname{críticos}^{42}$. No entanto, a fisiopatologia dessa situação e o complexo trabalho dos sistemas de defesa antioxidantes, têm limitado uma melhor visualização e definição dessa terapêutica. Algumas das intervenções sugeridas podem ser vislumbradas no Quadro 1.

Nathens et al. ${ }^{42}$ avaliaram 595 pacientes cirúrgicos críticos em um ensaio clínico randomizado com a suplementação de ácido ascórbico e $\alpha$-tocoferol, a qual resultou na redução da incidência de falência orgânica e de morbidade pulmonar, além de uma tendência na redução da mortalidade. Esses benefícios traduziram-se em menor tempo de ventilação mecânica, redução dos dias de internação na Unidade de Terapia Intensiva (UTI) e nenhum efeito adverso referente à suplementação.

Em outro estudo foi avaliada a utilização de um suplemento nutricional $(500 \mathrm{~mL}-250 \mathrm{kcal})$ enriquecido com antioxidantes (glutamina - 30g, vitamina $C-1500 \mathrm{mg}$, vitamina $\mathrm{E}-500 \mathrm{mg}, \beta$ caroteno $-10 \mathrm{mg}$, zinco - $20 \mathrm{mg}$ e selênio - $300 \mu \mathrm{g}$ ), com 14 pacientes cirúrgicos, durante 4 dias, via jejunostomia. No primeiro dia do pós-operatório houve redução significativa dos níveis plasmáticos de vitamina $C$, selênio, zinco e $\alpha$-tocoferol com valores abaixo da normalidade. Entre $\circ 1^{\circ}$ e $5^{\circ}$ dia após a cirurgia, houve um aumento significativo para todos os nutrientes, exceto para o $\beta$-caroteno. De acordo com os autores, essa diminuição dos níveis plasmáticos no pós-operatório imediato, resultou em parte da resposta inflamatória persistente refletida pelos valores plasmáticos elevados da proteína $C$ reativa. Além disso, a ausência dos efeitos no TBARS (substâncias reativas ao ácido tiobarbitúrico que indicam peroxidação lipídica) e GSPx (enzima glutationa peroxidase), é um argumento a favor da abordagem de suplementação combinada, via enteral ou parenteral, de micronutrientes em triagens futuras ${ }^{43}$.

Ao comparar grupos de ratos controle e queimados suplementados ou não com diferentes antioxidantes (vitamina C e E), Rao et al..$^{37}$ observaram diminuição no estresse oxidativo e epitelização mais rápida nos grupos suplementados.

Cetinkale et al. ${ }^{44}$ investigaram o efeito da terapia antioxidante na imunossupressão em ratos com 30\% de superfície corporal queimada. Os antioxidantes foram administrados por uma semana. Setenta e sete animais foram divididos em 7 grupos, sendo: G1 (controle) - sem queimaduras; G2 (queimados e sem suplementação); e os demais grupos receberam: $\mathrm{G} 3$ - allopurinol (inibidor de xantina oxidase); G4 - desferroxamina; G5 - polietileno glicol-catalase (PEG-CAT); G6 - N-acetilcisteína (NAC) e G7 - vitamina C.

Os autores demonstraram uma melhora significante da imunodepressão induzida pela 
698 | E. BARBOSA et al.

Quadro 1. Análise de estudos de intervenção com suplementação de antioxidantes em humanos e em animais em estado crítico.

\begin{tabular}{|c|c|c|c|c|c|}
\hline $\begin{array}{l}\text { Estudo (autores, } \\
\text { local, ano) }\end{array}$ & População & Suplemento utilizado & Quantidade & Tempo & Efeito/conclusão \\
\hline $\begin{array}{l}\text { Galley et al. } \\
(1997)^{45} \\
\text { Reino Unido }\end{array}$ & $\begin{array}{l}30 \text { pacientes } \\
\text { UTI com choque } \\
\text { séptico } \\
\text { Idade média }=67 \\
\text { anos }\end{array}$ & $\begin{array}{l}\text { NAC+ácido ascórbico }+\alpha- \\
\text { tocoferol ou placebo }(E V)\end{array}$ & $\begin{array}{l}\text { NAC } 150 \mathrm{mg} / \mathrm{kg} \text { por } 30^{\prime} \\
\text { seguido de } 20 \mathrm{mg} / \mathrm{kg} / \mathrm{h} \\
\text { de } 1 \mathrm{~g} \text { de ácido ascórbico } \\
\text { e } 400 \mathrm{mg} \text { de } \alpha \text {-tocoferol. }\end{array}$ & Dose única & $\begin{array}{l}\text { No grupo experimental houve } \uparrow \text { vi- } \\
\text { tamina C e do nitrito total no plas- } \\
\text { ma, a capacidade antioxidante to- } \\
\text { tal não alterou, a peroxidação } \\
\text { lipídica não alterou e o IRVS } \downarrow \text { nos } \\
120 \text { '. A administração de AOX este- } \\
\text { ve associada com mudanças hemo- } \\
\text { dinâmicas benéficas. }\end{array}$ \\
\hline $\begin{array}{l}\text { Berger et al. } \\
(1998)^{29} \\
\text { Suíça }\end{array}$ & $\begin{array}{l}20 \text { pacientes } \\
\text { queimados com } \\
\text { SCQ }>30 \% \\
\text { Idade média }=40 \\
\text { anos }\end{array}$ & $\begin{array}{l}\text { Suplementação de } \\
\text { elementos traços (ET) ou } \\
\text { ET padrão (controle). }\end{array}$ & 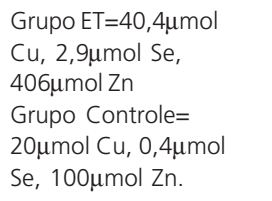 & 8 dias & $\begin{array}{l}\text { A suplementação de elementos tra- } \\
\text { ços parece ser benéfica após gran- } \\
\text { des queimaduras. Esteve associa- } \\
\text { da com } \downarrow \text { significante do } n^{\circ} \text { de bron- } \\
\text { copneumonia e menor tempo de } \\
\text { hospitalização. }\end{array}$ \\
\hline $\begin{array}{l}\text { Cetinkale et al. } \\
(1999)^{44} \\
\text { Turquia }\end{array}$ & $\begin{array}{l}77 \text { ratos Wistar } \\
\text { queimados com } \\
\text { SCQ=30\% com } \\
\text { espessura total } \\
\text { divididos em } 7 \\
\text { grupos }\end{array}$ & $\begin{array}{l}\text { 1०: não queimado-sem aox; } \\
2^{\circ}:(Q) \text { controle-sem aox; } \\
3^{\circ}:(Q) \text { Allopurinol (A); } \\
4^{\circ}:(Q) \text { Desferioxami-ne (D); } \\
5^{\circ}:(Q) \text { PEG-catalase (PEG- } \\
\text { CAT); 6 } 6^{\circ} \text { (Q) N-acetilcistína } \\
\text { (NAC); } \\
7^{\circ}:(Q) \text { Vitamina C (C) }\end{array}$ & $\begin{array}{l}A=50 \mathrm{mg} / \mathrm{kg} / \mathrm{d} \\
D=15 \mathrm{mg} / \mathrm{kg} / \mathrm{d} \\
P E G-C A T=1200 \mathrm{U} / \mathrm{kg} / \mathrm{d} \\
\mathrm{NAC}=1 \mathrm{mg} / \mathrm{kg} / \mathrm{d} \\
C=0,5 \mathrm{mg} / \mathrm{kg} / \mathrm{d}\end{array}$ & $\begin{array}{c}7 \text { dias } \\
\text { após lesão }\end{array}$ & $\begin{array}{l}\text { Houve melhora da imunidade celu- } \\
\text { lar com a utilização desses AOX, } \\
\text { significativamente com A e PEG-CAT, } \\
\text { sugerindo que em grandes queima- } \\
\text { dos os RL induzem a imunosupres- } \\
\text { são.Concluem que a intervenção } \\
\text { com AOX pode restaurar a imunida- } \\
\text { de celular. }\end{array}$ \\
\hline $\begin{array}{l}\text { Rao et al. } \\
(2001)^{37} \\
\text { India }\end{array}$ & $\begin{array}{l}50 \text { ratos com } \\
\text { queimadura de } \\
\text { profundidade } \\
\text { parcial divididos } \\
\text { em } 5 \text { grupos }\end{array}$ & $\begin{array}{l}\text { 1०: não queimado }(\mathrm{Q}) ; \\
2^{\circ}:(\mathrm{Q}) \text { controle; } \\
3^{\circ}:(\mathrm{Q}) \text { metronidazol; } \\
4^{\circ}:(\mathrm{Q}) \text { vitamina } \mathrm{C} ; \\
5^{\circ}:(\mathrm{Q}) \text { vitamina } \mathrm{E} \text {. }\end{array}$ & $\begin{array}{l}\text { 180mg/kg para cada } \\
\text { suplementação }\end{array}$ & Dose única & $\begin{array}{l}\text { A suplementação com metronidazol } \\
\text { e AOX, } \downarrow \text { MDA e acelerou a epite- } \\
\text { lização. }\end{array}$ \\
\hline $\begin{array}{l}\text { Nathens et al. } \\
(2002)^{42} \\
\text { EUA }\end{array}$ & $\begin{array}{l}595 \text { pacientes } \\
\text { UTI trauma ou } \\
\text { cirurgia } \\
\text { Idade média= } \\
39 \text { anos }\end{array}$ & $\begin{array}{l}\alpha \text {-tocoferol e ácido ascór- } \\
\text { bico (grupo experimental) ou } \\
\text { cuidado padrão (grupo con- } \\
\text { trole). }\end{array}$ & $\begin{array}{l}\alpha \text {-tocoferol } \\
1000 U \mathrm{Ul}(20 \mathrm{~mL}), \\
\text { via SNG a cada } 8 \mathrm{~h} \\
\text { ácido do ascórbico } \\
1000 \mathrm{mg}(100 \mathrm{~mL}), \\
\text { EV a cada } 8 \mathrm{~h}\end{array}$ & $\begin{array}{l}\text { Tempo de } \\
\text { duração na } \\
\text { UTI ou } 28 \\
\quad \text { dias }\end{array}$ & $\begin{array}{l}\text { A suplementação precoce utilizan- } \\
\text { do } \alpha \text {-tocoferol e ácido ascórbico em } \\
\text { pacientes críticos cirúrgicos, redu- } \\
\text { ziu a incidência de falência orgâni- } \\
\text { ca, o tempo de ventilação mecâni- } \\
\text { ca e o tempo de permanência na } \\
\text { UTI. Além disso, as altas doses não } \\
\text { causaram efeito adverso. }\end{array}$ \\
\hline $\begin{array}{l}\text { Berger et al. } \\
(2002)^{43} \\
\text { Suíça }\end{array}$ & $\begin{array}{l}12 \text { pacientes } \\
\text { cirúrgicos } \\
\text { Idade média= } \\
62 \text { anos }\end{array}$ & $\begin{array}{l}\text { Fórmula enteral: } 250 \mathrm{kcal} \text { e } \\
500 \mathrm{~mL} \text {, com: glutamina, se- } \\
\text { lênio, zinco, vitamina } C \text {, vi- } \\
\text { tamina E e } \beta \text {-caroteno. }\end{array}$ & $\begin{array}{l}30 \mathrm{~g} \text { glutamina } \\
300 \mathrm{~g} \text { selênio } \\
20 \mathrm{mg} \text { zinco } \\
1,5 \mathrm{~g} \text { vitamina } \mathrm{C} \\
500 \mathrm{mg} \text { vitamina } \mathrm{E} \\
10 \mathrm{mg} \beta \text {-caroteno }\end{array}$ & $\begin{array}{l}1 \text { dose - } 5 \\
\text { dias via } \\
\text { enteral }\end{array}$ & $\begin{array}{l}\text { Produto seguro e bem tolerado. } \\
\text { Houve } \downarrow \text { níveis plasmáticos de AOX } \\
\text { no } 1^{\circ} \text { P.O. Entre o } 1^{\circ} \text { e } 5^{\circ} \text { houve } \uparrow \\
\text { significante de todos micronutrientes } \\
\text { (exceto } \beta \text {-caro-teno). A ausência de } \\
\text { efeitos no TBARS e GPx é indicativo } \\
\text { favorável da suplementação com } \\
\text { AOX. }\end{array}$ \\
\hline $\begin{array}{l}\text { Long et al. } \\
(2003)^{50} \\
\text { EUA }\end{array}$ & $\begin{array}{l}12 \text { pacientes } \\
\text { criticamente } \\
\text { doente }\end{array}$ & Vitamina C (EV) & $\begin{array}{l}300 \mathrm{mg} / \mathrm{dia} \\
1000 \mathrm{mg} / \mathrm{dia} \\
3000 \mathrm{mg} / \mathrm{dia}\end{array}$ & $\begin{array}{l}2 \text { dias } \\
2 \text { dias } \\
2 \text { dias }\end{array}$ & $\begin{array}{l}\text { A concentração inicial da vitamina } \\
\text { C inicial estava muito baixa e um } \uparrow \\
\text { significativo só foi observado após } \\
\text { suplementação com } 3000 \mathrm{mg} / \mathrm{d} \text {, in- } \\
\text { dicando grande consumo e neces- } \\
\text { sidade de suplementação nesta po- } \\
\text { pulação. }\end{array}$ \\
\hline $\begin{array}{l}\text { Barbosa } \\
(2006)^{51} \\
\text { Brasil }\end{array}$ & $\begin{array}{l}32 \text { pacientes } \\
\text { pediátricos } \\
\text { queimados } \\
\text { Idade média= } \\
54 \text { meses } \\
\text { Média SCQ= } \\
16 \%\end{array}$ & $\begin{array}{l}\text { Grupo estudo: } \mathrm{n}=17 \\
\text { Vitamina } \mathrm{C} \\
\text { Vitamina } \mathrm{E} \\
\text { Zinco } \\
\text { Grupo controle: } \mathrm{n}=15 \\
\text { xaropes com sabor e cor se- } \\
\text { melhantes }\end{array}$ & $\begin{array}{l}1,5 \mathrm{UL} / \mathrm{dia} \\
1,3 \mathrm{UL} / \mathrm{dia} \\
2 \times \mathrm{RDA} / \mathrm{dia}\end{array}$ & 7 dias & $\begin{array}{l}\text { A suplementação } \downarrow \text { significativamen- } \\
\text { te o tempo de reepitelização, além } \\
\text { de proporcionar uma } \mathrm{PL}<\text {, e uma } \\
\text { redução da capacidade antioxi- } \\
\text { dante total estatisticamente }<\text { no } \\
\text { grupo estudo. Apesar de não signifi- } \\
\text { cante, o tempo de hospitalização, } \\
\text { o número e os dias de antibióticos/ } \\
\text { paciente foram inferiores para o } \\
\text { grupo suplementado. }\end{array}$ \\
\hline
\end{tabular}

NAC: N-acetilcisteína; EV: endovenoso; IRVS: índice de resistência vascular sistêmica; AOX: antioxidante; Q: queimado; SCQ: superfície corporal queimada; RL: radical livre; MDA: malondialdeído; SOD: superóxido dismutase; UTI: Unidade de terapia intensiva; GSH: glutationa; TBARS: ácido tiobarbitúrico; DMOS: disfunção múltipla de órgãos e sistemas; GPx: glutationa peroxidase; PL: peroxidação lipídica. 
queimadura refletida na resposta de hipersensibilidade de contato, com todos os antioxidantes utilizados. Em relação à reação hospedeiro versus enxerto, o tratamento com allopurinol e PEG-CAT mostrou uma melhora significativa, enquanto que a desferroxamina, a NAC e a vitamina C demonstraram melhora, porém sem significância. Com esses dados concluiu-se que a queimadura foi profundamente imunossupressiva e a intervenção precoce com terapia antioxidante foi capaz de influenciar de forma positiva na restauração da imunidade celular.

Uma outra pesquisa avaliou o efeito de antioxidantes intravenosos no status de antioxidantes, na peroxidação lipídica e no óxido nítrico em relação aos parâmetros hemodinâmicos em pacientes com choque séptico. Trinta pacientes foram randomizados em GE (experimental), para receber antioxidantes (n-acetilcisteína-NAC, ácido ascórbico e $\alpha$-tocoferol) e grupo GC (controle) para receber dextrose. O nível basal da vitamina $C$ estava baixo em todos os pacientes. Nos 16 pacientes recebendo antioxidantes, a vitamina $C$ aumentou $(p=0,0002)$, mas a capacidade antioxidante total não foi alterada. Os peróxidos lipídicos estavam elevados em ambos os grupos, mas não aumentaram significantemente nos suplementados. O nitrito total plasmático aumentou $(p=0,007)$ e os índices cardíacos se elevaram nos 60 e 120 minutos de forma significativa no GE. Além disso, o índice de resistência vascular sistêmica diminuiu nos pacientes tratados com antioxidantes. Os autores concluíram que esta melhora dos parâmetros hemodinâmicos justificariam a terapêutica coadjuvante de antioxidantes no manejo de pacientes com choque séptico ${ }^{45}$.

Mingjian et al. ${ }^{46}$, de forma semelhante, avaliaram pacientes queimados $(n=35)$, dos quais 18 receberam vitamina $E(100 \mathrm{mg} / \mathrm{dia})$ por, pelo menos, 16 dias. Os autores evidenciaram que a suplementação aumentou a concentração sangüínea da mesma, apesar de se ter mantido abaixo dos valores normais. Além disso, os níveis sangüíneos de peróxidos lipídicos foram reduzidos.
Chai et al ${ }^{47}$ demonstraram efeito protetor da suplementação com vitamina $\mathrm{E}$ em pacientes severamente queimados, indicando que essa vitamina age como um eficiente removedor de radicais livres e protege a função dos neutrófilos em conseqüência do aumento dos valores da SOD e diminuição do MDA, além da restauração do prejuízo da função dos neutrófilos.

Com esses resultados, durante a queimadura o $\alpha$-tocoferol parece ter um efeito protetor na estabilização da membrana pela doação de seu átomo de hidrogênio para o radical de oxigênio, formando o radical tocoferil e, dessa forma, reduz a peroxidação lipídica. Por isso, o $\alpha$-tocoferol parece ser crucial como agente de defesa celular contra o ataque oxidativo ${ }^{48}$.

O aumento de radicais livres parece comprometer os níveis de antioxidantes em paciente críticos e afetar adversamente sua recuperação. Nesse sentido, Miyagatami et al. ${ }^{49}$ demonstraram que altas doses de ácido ascórbico resultaram em $80 \%$ de sobreviventes $(p=0,05)$ de ratos sépticos comparados com $50 \%$ sem ácido ascórbico. Também observaram o aumento da glutationa hepática, o principal removedor de radical livre intracelular, no grupo suplementado.

Long et al. ${ }^{50}$ avaliaram os níveis de vitamina C plasmático em pacientes criticamente doentes $(n=12)$. Os resultados demonstraram que a concentração inicial de vitamina $C$ estava muito baixa e não respondeu com 300 $\mathrm{mg} / \mathrm{d}$ de suplementação e apenas alcançou níveis normais limítrofes após dois dias com 1000mg/d. Um aumento significativo só foi observado após a suplementação com $3000 \mathrm{mg} / \mathrm{d}$. No entanto, apesar do aumento dos níveis plasmáticos com essa megadose, parece que o pool de vitamina $C$ não saturou, uma vez que nem toda a vitamina $C$ foi excretada na urina.

Em um ensaio clínico randomizado duplo cego com crianças queimadas ( $n=32$ ), a suplementação de vitamina $C$, vitamina $E$ e zinco reduziu significantemente o tempo de reepitelização, além de proporcionar uma peroxidação lipídica e uma redução da capacidade antioxidante total 
estatisticamente menor no grupo estudo. Além disso, apesar de não significante, o tempo de hospitalização, o número e os dias de antibióticos/ paciente foram inferiores para o grupo estudo, apesar da maior ocorrência do número de enxertos/paciente para essas mesmas crianças. Segundo a autora, embora os resultados com a utilização de suplementos antioxidantes combinados tenham sido promissores, outros estudos bem controlados com pacientes queimados necessitam ser realizados para obtenção de evidências sólidas de sua eficácia ${ }^{51}$.

De acordo com Galley et al. ${ }^{45}$, a mais efetiva forma de suplementação de antioxidante é, provavelmente, incluir combinações dos mesmos com ações sinérgicas conhecidas. A vitamina $E$ protege contra a peroxidação lipídica e é considerada, biologicamente, o antioxidante mais importante. A vitamina C é o mais poderoso doador de elétron, reagindo com os radicais superóxidos e hidroxila com capacidade de proteger tanto os componentes do citoplasma como as membranas celulares. No citoplasma, o ascorbato age como antioxidante direto, ou seja, como scavenger para os radicais livres gerados durante o metabolismo celular. Já nas membranas celulares, o ascorbato age como um antioxidante indireto, reduzindo o radical $\alpha$-tocoferil a $\beta$-tocoferol ${ }^{52}$.

\section{O N CLUSÃ O}

Os vários trabalhos publicados até o momento evidenciam a participação dos radicais livres no prolongamento da injúria. Ao mesmo tempo, cada vez mais, compreende-se que os antioxidantes podem promover uma proteção para esse prejuízo; muito embora sejam poucos os trabalhos prospectivos e controlados que demonstrem conclusivamente os efeitos da suplementação na morbidade e mortalidade dos pacientes queimados. Além disso, há escassez de pesquisas em subgrupos como idosos e crianças. Justificam-se, portanto, novos protocolos que não somente corroborem os fatos delineados, como os expandam para populações específicas.
A terapia antioxidante parece promissora em atenuar os efeitos da produção descontrolada de radicais livres em pacientes graves, acarretando a possível diminuição do estresse oxidativo e, conseqüentemente, a redução do risco de complicações infecciosas, melhor cicatrização, além do menor tempo de permanência hospitalar. Porém, o uso de um nutriente de forma isolada deve ser avaliado cuidadosamente, em conseqüência do sinergismo que existe entre eles e pelo risco de efeito oxidante paradoxal com altas doses de um único nutriente.

\section{COLABORADORES}

E. BARBOSA desenvolveu a metodologia e elaborou o artigo. E.A.M. MOREIRA contribuiu na elaboração e revisão crítica do artigo. J. FAINTUCH e M.J.L. PEREIMA contribuíram na revisão crítica do artigo.

\section{REFERÊ NCIAS}

1. Stuart JD, Kenney JG, Morgan RF. Pediatrics burns. Am Farm Physician. 1987; 36(4):139-46.

2. Harmel RP, Vane DW, King DR. Burn care in children: special considerations. Clin Plast Surg. 1985; 13(1): 95-105.

3. Deitch EA, Dobke M. Failure of local imunity: a potencial cruse of burn wound sepsis. Arch Surg. 1984; 120(1):78-84.

4. Latha $B, B a b u M$. The involvement of free radicals in burn injury: a review. Burns. 2001; 27(4):309-17.

5. Demuth MWE. Advanced burn life support: manual. In: International society for burns injuries. Edinburgh: ISBI; 2001. p.86.

6. Yoshida WB. Radicais livres na síndrome da isquemia e reperfusão. Cir Vasc Angiol. 1996; 1282-95.

7. Horton JW. Free radicals and lipid peroxidation mediaded injury in burn trauma: the role of antioxidant therapy. Toxicol. 2003; 189(1-2):75-88.

8. Granger DN. Role of xanthine oxidase and granulocytes in ischemia-reperfusion injury. Am J Physiol. 1988; 255(6):1269-75.

9. Demling $\mathrm{RH}$, La Londe C. Systemic lipid peroxidation and inflammation induced by thermal injury persist into post-resuscitation period. J Trauma. 1990; 30(1):69-74. 
10. Berger MM, Chiolero R. Relations between cooper, zinc and selenium intakes and malondialdehyde excretion after major burns. Burns. 1995; 21(7): 507-12.

11. Cetinkale O, Belce A, Konukoglu D, Senyuva C, Gumustas MK, Tas T. Evaluation of lipid peroxidation and total antioxidant status in plasma of rats following termal injury. Burns. 1997; 23(2): 114-6.

12. Bertin-Maghit M, Goudable J, Dalmas E, Steghens JP, Bouchard C, Gueugniaud PY, et al. Time course of oxidative stress after major burns. Intensive Care Med. 2000; 26 (6):800-3.

13. Haycock JW, Ralston DR, Morris B, Freedlander E, MacNeil S. Oxidative damage to protein and alterations to antioxidant levels in human cutaneous thermal injury. Burns. 1998; 23(7/8): 533-40.

14. Pintaudi AM, Tesoriere L, D'Arpa N, D'Amelio L, $D^{\prime}$ arpa $D$, Bongiorno $A$, et al. Oxidative stress after moderate to extensive burning in humans. Free Radic Res. 2000; 33(2):139-46.

15. Correia MITD. Antioxidação: o papel das vitaminas. Rev Bras Nutr Clin. 2001; 16(2):74-8.

16. Sies H. Strategies of antioxidant defence. Review. Eur J Bioch. 1993; 215(2):213-9.

17. Leite HP, Sarni RS. Radicais livres, antioxidantes e nutrição. Rev Bras Nutr Clin. 2003,18(2):60-5.

18. Evans P, Halliwell B. Micronutrients: oxidant, antioxidant status. Br J Nutr. 2001; 85(2):S67-S74.

19. Goode HF, Cowley HC, Waler BE. Decreased antioxidant status and increased lipid peroxidation in patients with septic shock and secondary organ dysfunction. Crit Care Med. 1995; 23 (4):646-51.

20. Stahl W. Lipid oxidation and antioxidants. Curr Opin Clin Nutr Metab Care. 2000; 3(2):121-6

21. Bird RP, Draper HH. Comparative studies on different methods of malonaldehyde determination. Methods Enzymol. 1984; 105:299-305.

22. Ben Baouali A, Aube $H$, Maupoil V, Blettery B, Rochette L. Plasma lipid peroxidation in critically ill patients: importance of mechanical ventilation. Free Radic Biol Med. 1994; 16(2):223-7.

23. Lalonde C, Nayak U, Hennigan J, Demling RH. Excessive liver oxidant stress causes mortality in response to burn injury combined with endotoxin and is prevented with antioxidants. J Burn Care Rehabil. 1997; 18(3):187-92.

24. Bulkley GB. The role of oxygen free radical in human disease processes. Surgery. 1983; 94(3): 407-13.

25. Chance B, Sies $H$, Boveris A. Hydroperoxide metabolism in mammalian organs. Physiol Res. 1979; 59(3):527-602.
26. Papas AM. Diet and antioxidant status. Food Chem Toxicol. 1999; 37(9-10):999-1007.

27. Leite HP, Sarni RS. Radicais livres, antioxidantes e nutrição. Rev Bras Nutr Clin. 2003; 18(2):60-5.

28. Jeschke MG, Barrow RE, Mlcak RP, Herndon DN. Endogenous anabolic hormones and hypermetabolism. Effect of traum and gender differences. Ann Surg. 2005; 241(5):759-68.

29. Berger MM, Spertini F, Shenkin A, Wardle C, Wiesner $L$, Schindler $C$, et al. Trace element supplementation modules pulmonary infection rates after major burns: a double-bind, placebo-controlled trial. Am J Clin Nutr. 1998; 68(2): 365-71.

30. Chandra RK, McBean LD. Zinc and immunity. Nutrition. 1994; 10(1):79-80.

31. National Research Council (USA). Recommended dietary allowances. Washington (DC): National Academy Press; 1989

32. Berger MM, Cavadini C, Chiolero R. Guinchard S, Krupp S, Dirren H. Influence of large intakes of trace elements on recovery after major burns. Nutrition. 1994; 10(4):327-34.

33. Institute of Medicine. DRIs: dietary reference intakes for vitamin A, vitamin K, arsenic, boron, chromium, cooper, iodine, iron, manganese, molybdenum, nickel, silicon, vanadium, and zinc. Washington (DC): National Academy Press; 2002.

34. Voruganti VS, Klein GL, Lu HX, Thomas S, Graves JHF, Herndon DN. Impaired zinc and cooper status in children with burn injuries: need to reassess nutritional requeriments. Burns. 2005; 31(6): 711-6.

35. Agay D, Anderson RA, Sandre C, Bryden NA, Alonso $A$, Roussel AM, et al. Alterations of antioxidant trace elements $(\mathrm{Zn}, \mathrm{Se}, \mathrm{Cu})$ and related metalloenzymes in plasma and tissues following burn injury in rats. Burns. 2005; 31(3):366-71.

36. Goode HF, Webster NR. Free radicals and trace element metabolism in sepsis and injury. $\mathrm{Br} J$ Intensive Care. 1992; 2312-22.

37. Rao MC, Ghosh A, Raghothama C, Bairy KL. Does metronidazole reduce lipid peroxidation in burn injuries to promote healing? Burns. 2002; 28(5): 427-9.

38. Gey KF. Vitamins E plus C and interacting conutrients required for optimal health. Biofactors. 1998; 7(1/2):113-74.

39. Hemila H. Vitamin C intake and susceptibility to pneumonia. Pediatr Infect Dis. 1997; 16(9):836-7.

40. Meydani SN, Meydani M, Blumberg JB, Leka LS, Siber G, Loszewski $R$, et al. Vitamin $E$ supplementation and in vivo immune response in 
health elderly subject: a randomized controlled trial. JAMA. 1997; 277(17):1380-6.

41. Berger MM. Can oxidative damage be treated nutritionally? Clin Nutr. 2005; 24(2):172-83.

42. Nathens AV, Neff MJ, Jurkovich GJ, Klotz P, Farver K, Ruzinski JT, et al. Randomized, prospective trial of antioxidant supplementation in critically ill surgical patients. Ann Surg. 2002; 236(6):814-22.

43. Berger MM, Goette J, Stehle P, Cayeux MC, Chiolero R, Schroeder J. Enteral absorption of a solution with high dose antioxidants and glutamine after upper gastrointestinal surgery. Clin Nutr. 2002; 1:S073.

44. Cetinkale O, Senel O, Bulan R. The effect of antioxidant therapy on cell-mediated immunity following burn injury in animal model. Burns. 1999; 25(2):113-8.

45. Galley H, Howdle PD, Walker BE, Webster NR. The effects of intravenous antioxidants in patients with septic shock. Free Radic Biol Med. 1997; 23(5): 768-74

46. Mingjian Z, Qifang W, Lanxing G, Hong J, Zongyin $W$. Comparative observation of the changes in serum lipid peroxides influenced by the supplementation of vitamin $\mathrm{E}$ in burn patients and healthy controls. Burns. 1992; 18(1):19-21.

47. Chai J, Guo Z, Sheng Z. Protective effects of vitamin $E$ on impaired neutrophil phagocyte function in pacients with severe burn. Chung Hua Cheng Hsing Shao Shang Wai Ko Tsa Chih. 1995; 11(1): 32-5.

48. Burton GW, Joyce A, Ingold $\mathrm{KU}$. Is vitamin $\mathrm{E}$ the only lipid-soluble, chain breaking antioxidant in human blood plasma and erythrocyte membranes? Arch Biochem Biophys. 1983; 221(1):281-90.

49. Miyagatani $Y$, Rounds JD, Chambers EA, Robinson MK, Jacobs DO. High-dose vitamin C enhances hepatic glutathione levels and increases survival of septic rats. Surg Forum 1998; 49:55-9.

50. Long CL, Maull Kl, Krishnan RS, Laws HL, Geiger $\mathrm{BS}$, Borghesi $\mathrm{L}$, et al. Ascorbic acid dynamics in the seriously ill and injured. J Surg Res. 2003; 109(2): 144-8.

51. Barbosa E. Efeito da suplementação de vitamina E, vitamina $C$ e zinco no estresse oxidativo e no tempo de reepitelização em pacientes pediátricos queimados [dissertação]. Florianópolis: Universidade Federal de Santa Catarina; 2006.

52. May JM, Qu ZC, Whitesell RR. Ascorbic acid recycling enhances the antioxidant reserve of human erythrocytes. Biochemistry. 1995; 34(39): 12721-8.

Recebido em: 13/9/2005

Versão final reapresentada em: 30/10/2006 Aprovado em: 27/7/2007 